\title{
Qualitative and Quantitative Analysis of Pharmaceutical Compounds by MALDI-TOF Mass Spectrometry
}

\author{
Jeroen J. A. van Kampen, ${ }^{\mathbf{t} \neq}$ Peter C. Burgers, ${ }^{\dagger}$ Ronald de Groot, $^{\S}$ and Theo M. Luider*.† \\ Department of Neurology, Laboratory of Neuro-Oncology, and Department of Pediatrics, Division of Pediatric Infectious \\ Diseases \& Immunology, Sophia Children's Hospital, Erasmus Medical Center, Rotterdam, The Netherlands, and \\ Department of Pediatrics, University Medical Center St. Radboud, Nijmegen, The Netherlands
}

In this report, we discuss key issues for the successful application of MALDI-TOF mass spectrometry to quantify drugs. These include choice and preparation of matrix, nature of cationization agent, automation, and data analysis procedures. The high molecular weight matrix mesotetrakis(pentafluorophenyl)porphyrin eliminates chemical noise in the low-mass range, a "brushing" spotting technique in combination with prestructured target plates enables fast preparation of homogeneous matrix crystals, and addition of $\mathrm{Li}^{+}$leads to intense cationized drug species. Complex biological samples were cleaned up using a 96-well solid-phase extraction plate, and the purified samples were automatically spotted by a pipetting robot. To obtain a suitable data analysis procedure for the quantitative analysis of drugs by MALDI-TOF mass spectrometry, various data processing parameters were evaluated on our two model drugs lopinavir and ritonavir. Finally, and most importantly, it is shown that the abovedescribed procedure can be successfully applied to quantify clinically relevant concentrations of lopinavir, an HIV protease inhibitor, in extracts of small numbers of peripheral blood mononuclear cells $\left(1 \times 10^{6}\right)$.

In our pediatrics departments, we need a fast, reliable, and sensitive method to quantify antiretroviral drugs in various biological samples, such as extracts from peripheral blood mononuclear cells (PBMCs). To this end, we have initiated a study to test the suitability of matrix-assisted laser desorption/ionization time-of-flight (MALDI-TOF) mass spectrometry for such clinical applications.

MALDI and electrospray ionization (ESI) are the two most extensively used ionization techniques for the mass spectrometric analysis of biological and biomedical compounds. However, only ESI is commonly used for qualitative and quantitative analysis of low molecular weight compounds (<1000 Da) such as drugs. This is because, in contrast to MALDI, ESI does not require the addition of matrix molecules; such matrix molecules may undergo association reactions leading to interference peaks in the low-mass

* Corresponding author. Telephone: +31 10 4638069. Fax: +31 104088365 . E-mail: t.luider@erasmusmc.nl.

$\dagger$ Department of Neurology, Erasmus Medical Center.

* Department of Pediatrics, Erasmus Medical Center.

$\S$ University Medical Center st. Radboud. range. Nevertheless, MALDI offers some specific advantages over ESI. Perhaps the most important distinction of MALDI is that it is capable of very high sample throughput with minimum instrument maintenance. In addition, MALDI is less susceptible to ion suppression. It is for these reasons that in recent years MALDI has seen its emergence in the field of small-molecule analysis. ${ }^{1}$

Two major phenomena hamper the application of the MALDI technique to the analysis of small molecules. As mentioned above, MALDI suffers from matrix-derived chemical interference in the low-mass range. ${ }^{2}$ From a quantitative perspective, MALDI suffers from poor reproducibility of the signal abundance. ${ }^{1}$

The molecular masses of conventional MALDI matrixes range from 100 to $300 \mathrm{Da}$. Due to ion/molecule reactions in the matrix plume, a large number of matrix-derived peaks are usually observed in the low-mass range. ${ }^{2}$ For example, the often used matrix 2,5-dihydroxybenzoic acid (DHB; $\left.\mathrm{C}_{7} \mathrm{H}_{6} \mathrm{O}_{4}\right)$ produces, after laser irradiation, the series of $\left(\mathrm{C}_{7} \mathrm{H}_{5} \mathrm{O}_{4} \mathrm{Na}\right)_{n} \mathrm{Na}^{+}$ions in admixture with other adducts, even in the presence of residual sodium ions. ${ }^{3}$ These abundant matrix-related peaks seriously hamper the qualitative and quantitative analysis of small molecules. One way to avoid such interference signals is to choose a matrix whose molecular weight is in excess of that of the analyte molecule. ${ }^{4,5}$ Meso-tetrakis(pentafluorophenyl)porphyrin (F20TPP) is such a high molecular MALDI matrix $(\mathrm{MW}=974.6 \mathrm{Da})$ and has successfully been used for the analysis of various small molecules, for example, poly(ethylene glycol)s, fatty acids, and sugars. ${ }^{6-8}$

A variety of approaches have been reported to enhance the signal reproducibility of MALDI mass spectrometers, and they can be divided into two main groups: (1) enhancement of homogeneous matrix crystallization. enhancement of homoge-

(1) Cohen, L. H.; Gusev, A. I. Anal. Bioanal. Chem. 2002, 373, 571-586.

(2) Krutchinsky, A. N.; Chait, B. T. L.Am. Soc. Mass Spectrom. 2002, 13, 129 134.

(3) Gouw, J. W.; Burgers, P. C.; Trikoupis, M. A.; Terlouw, J. K. Rapid Commun. Mass Spectrom. 2002, 16, 905-912.

(4) Chen, Y. T.; Ling, Y. C. I. Mass Spectrom 2002, 37, 716-730.

(5) Ling, Y. C.; Lin, L.; Chen, Y. T. Rapid Commun. Mass Spectrom. 1998, 12, 317-327.

(6) Ayorinde, F. O.; Bezabeh, D. Z.; Delves, I. G. Rapid Commun. Mass Spectrom. 2003, 17, 1735-1742.

(7) Ayorinde, F. O.; Hambright, P.; Porter, T. N.; Keith, Q. L., Jr. Rapid Commun. Mass Spectrom. 1999, 13, 2474-2479.

(8) Hlongwane, C.; Delves, I. G.; Wan, L. W.; Ayorinde, F. O. Rapid Commun. Mass Spectrom. 2001, 15, 2027-2034.

Analytical Chemistry, Vol. 78, No. 15, August 1, 20065403 
neous analyte incorporation into the matrix crystals, or both. This can be achieved, for example, by preparing matrix crystals using a fast evaporation protocol or by using prestructured target plates..$^{9,10}$ (2) averaging out variations in instrument response by employing an internal standard and by averaging out multiple spectra of one sample. ${ }^{11-13}$

In this study, a strategy is presented to overcome the two main limitations of MALDI-TOF mass spectrometry for the qualitative and quantitative analysis of small molecules. To reduce the number of matrix-derived peaks in the low-mass range, we have used a high molecular weight matrix, viz. F20TPP. To increase reproducibility (precision), we have developed, using prestructured target plates, a fast evaporation protocol for the matrix F20TPP. To increase reproducibility further, instrument response variation was minimized by using an internal standard, by averaging out 1000 spectra per sample, and by measuring each sample 4-fold. In addition, the effects of various data analysis procedures on the precision and accuracy of analyte abundances was investigated. Finally, we demonstrate that MALDI-TOF mass spectrometry can be successfully used for the quantitative analysis of lopinavir, an HIV protease inhibitor, in extracts of PBMCs.

\section{EXPERIMENTAL SECTION}

Pharmaceutical Compounds. Lopinavir and ritonavir were kindly provided by Abbott Laboratories. Indinavir was kindly supplied by Merck Sharp \& Dohme. Saquinavir was kindly provided by F. Hoffmann-La Roche. Nelfinavir was kindly provided by Pfizer Inc. Zidovudine, lamivudine, abacavir, and amprenavir were kindly provided by GlaxoSmithKline. Nevirapine and tipranavir were kindly provided by Boehringer Ingelheim. Erythromycin, omeprazole, carbamazepine, sulfamethoxazole, levofloxacin, primaquine, metoprolol, acetylsalicylic acid, amikacin, tobramycin, ampicillin, acetaminophen, trimethoprim, penicillin $\mathrm{G}$, and rifampicin were purchased from Sigma-Aldrich.

Chemicals. Lithium iodide, sodium iodide, potassium iodide, rubidium iodide, cesium iodide, HPLC grade water, and 5,10,15,20-tetrakis (pentafluorophenyl)porphyrin were obtained from SigmaAldrich. All organic solvents were obtained from J. T. Baker. All chemicals were purchased at the highest purity grade available and were used without further purification.

Standards for Qualitative Analysis. Dilution series for each pharmaceutical compound were prepared in ethanol/water (1:1). Dilution series A contained pharmaceutical compounds at a concentration of $8000,4000,2000,1000$, and $500 \mathrm{fmol} / \mu \mathrm{L}$. Dilution series B contained pharmaceutical compounds at a concentration of $80,40,20,10$, and $5 \mathrm{fmol} / \mu \mathrm{L}$. Samples were stored at $-20^{\circ} \mathrm{C}$.

Standards for Quantitative Analysis of Pure Lopinavir and Ritonavir. Calibrators were prepared of pure lopinavir and ritonavir at concentrations of 1000, 900, 800, 700, 500, 400, 300, $200,100,80,40,20,15,10,5$, and $1 \mathrm{fmol} / \mu \mathrm{L}$. Each calibrator was spiked with $50 \mathrm{fmol} / \mu \mathrm{L}$ indinavir (internal standard). All calibrators were prepared in ethanol/water $(1: 1)$ and stored at $-80^{\circ} \mathrm{C}$.

(9) Schuerenberg, M.; Luebbert, C.; Eickhoff, H.; Kalkum, M.; Lehrach, H.; Nordhoff, E. Anal. Chem. 2000, 72, 3436-3442.

(10) Vorm, O.; Roepstorff, P.; Mann, M. Anal. Chem. 1994, 66, 3281-3287.

(11) Duncan, M. W.; Matanovic, G.; Cerpa-Poljak, A. Rapid Commun. Mass Spectrom. 1993, 7, 1090-1094.

(12) Hatsis, P.; Brombacher, S.; Corr, J.; Kovarik, P.; Volmer, D. A. Rapid Commun. Mass Spectrom. 2003, 17, 2303-2309.

(13) Rideout, D.; Bustamante, A.; Siuzdak, G. Proc. Natl. Acad. Sci. U.S.A. 1993, 90, 10226-10229.
Standards for Quantitative Analysis of Lopinavir in PBMC Extracts. PBMCs were isolated from a buffy coat (Sanquin) using a Ficoll density gradient. Calibrators were prepared of pure lopinavir in ethanol/water (1:1) at concentrations of 4000, 3000, 2000, 1000, 400, 200, 100, 50, and $25 \mathrm{fmol} / \mu \mathrm{L}$ spiked with 500 $\mathrm{fmol} / \mu \mathrm{L}$ saquinavir (internal standard). A $100-\mu \mathrm{L}$ aliquot of each calibrator was added to a dry pellet of $1 \times 10^{6} \mathrm{PBMCs}$, and PBMCs were extracted overnight in these calibrator solutions at $5{ }^{\circ} \mathrm{C}$. Subsequently, cell debris was spun down and supernatants were loaded onto a 96-well solid-phase extraction plate (Oasis HLB $\mu$ elution plate, Waters). The loaded samples were washed twice with $200 \mu \mathrm{L}$ of methanol/water (1:19) and $200 \mu \mathrm{L}$ of methanol/ water (1:1), respectively. Samples were eluted from the solid-phase extraction plate using $100 \mu \mathrm{L}$ of methanol/water (3:1). The eluted samples were dried using a SpeedVac (Savant) and were stored at $-20{ }^{\circ} \mathrm{C}$. On the day of analysis, the dried samples were reconstituted in $100 \mu \mathrm{L}$ of ethanol/water (1:1).

Matrix and Matrix Spotting Technique. Four parts of 25 $\mathrm{mg} / \mathrm{mL}$ F20TPP, dissolved in 100\% acetone, were mixed with one part of $100 \mathrm{mM} \mathrm{LiI,} \mathrm{NaI,} \mathrm{KI,} \mathrm{RbI,} \mathrm{or} \mathrm{CsI.} \mathrm{LiI,} \mathrm{and} \mathrm{NaI,} \mathrm{dissolved}$ in $100 \%$ acetone, and $\mathrm{KI}, \mathrm{RbI}$, and CsI were dissolved in $100 \%$ methanol. Thus, the final matrix solutions contained $20 \mathrm{mg} / \mathrm{mL}$ F20TPP and $20 \mathrm{mM}$ alkali iodide. Matrix crystals were prepared on an AnchorChip target plate with $800-\mu \mathrm{m}$ sized hydrophilic anchors (Bruker Daltonics) by the following "brushing" technique: A $10-\mu \mathrm{L}$ pipet tip was filled with matrix solution. Next, the pipet was positioned such that the tip just touched the target plate above the first spot. Next, the plunger was slightly pressed and at the same time the pipet was quickly dragged downward over the target spots.

Sample Spotting. All samples were spotted in 4 -fold $(1 \mu \mathrm{L} /$ replicate) on top of the matrix crystals using a ClinProtRobot pipetting robot, which was controlled by ClinProtRobot Workflow, Editor version 1.0 software (Bruker Daltonics, Germany).

Sample Measurement. All experiments were performed on an Ultraflex MALDI-TOF/TOF mass spectrometer (Bruker Daltonics) equipped with a 50-Hz nitrogen laser (337 nm). Mass spectra were recorded in the positive ion reflectron mode. FlexControl version 2.4 software was used to operate the mass spectrometer. A sample spot containing matrix only was used to manually set the laser power $\sim 5 \%$ above the threshold for ionization. The samples containing the analytes were measured automatically using the AutoXecute part of the FlexControl software. Mass spectra were recorded by accumulating 50 shots on 20 different positions on each sample spot. No spectra were rejected (i.e., fuzzy control for spectra accumulation was not used). Four replicates of each sample were measured, and each experiment was repeated three times.

Data Analysis. A FlexAnalysis script, kindly provided by Bruker Daltonics, was used to process the raw mass spectra in batch and to pick all peaks for a specified $m / z$ range. Each mass spectrum was processed with and without baseline subtraction, and peak picking was performed using the "centroid" peak picking algorithm (monoisotope only) as well as the "SNAP" peak picking algorithm (all isotopes). Baseline subtraction was performed using the "median" baseline subtraction algorithm with 0.8 flatness. For the centroid peak picking algorithm, the signal-to-noise ratio (S/ $\mathrm{N})$ threshold was set at 3 times above the standard deviation of 


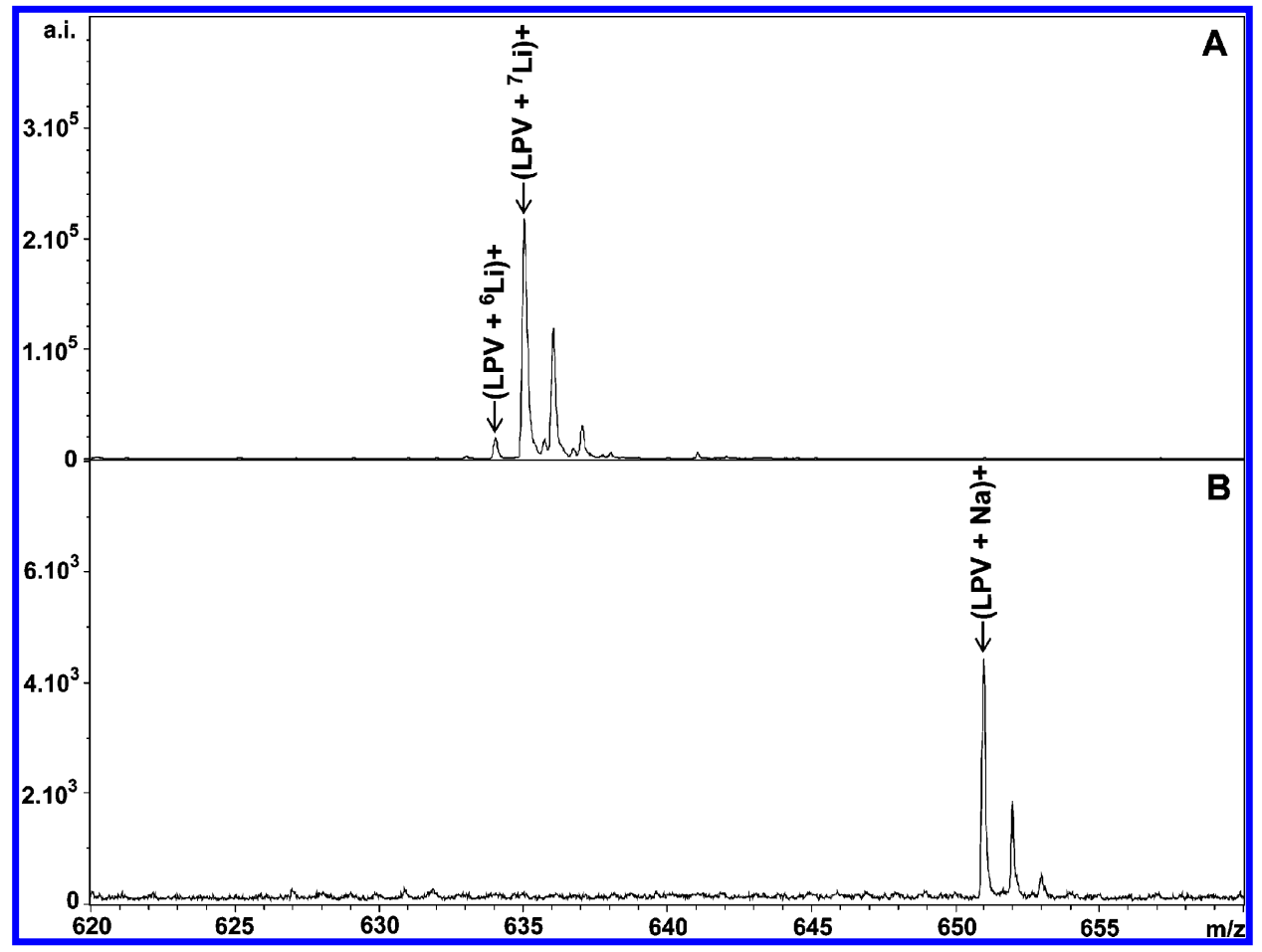

Figure 1. Analysis of lopinavir using F20TPP as matrix. a.i., absolute intensity. The molecular weight of lopinavir (LPV) is 628.8 Da. Panel A: An intense signal for lithiated lopinavir was observed when 1 pmol of lopinavir was analyzed with $20 \mathrm{mg} / \mathrm{mL}$ F20TPP $+20 \mathrm{mM}$ Lil. No protonated lopinavir was observed. Panel B: A signal was observed for lopinavir cationized by residual sodium ions when 1 pmol of lopinavir was analyzed with $20 \mathrm{mg} / \mathrm{mL}$ F20TPP. No protonated lopinavir was observed.

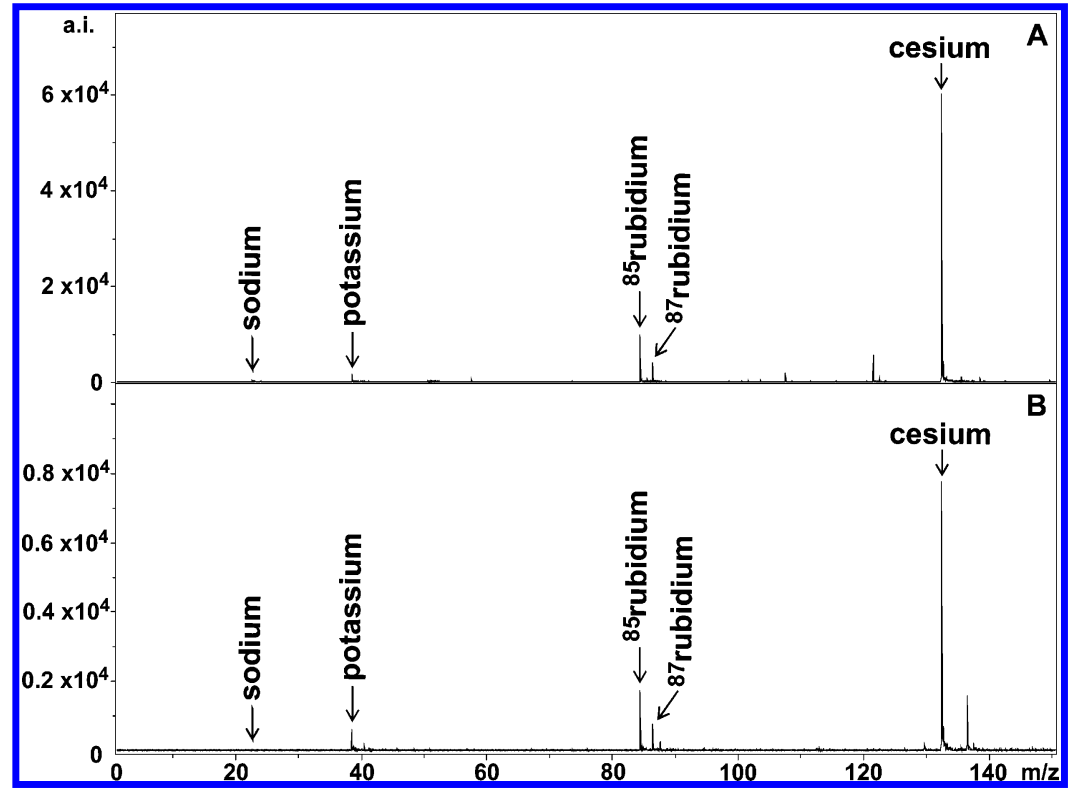

Figure 2. Abundances of free cations from equimolar alkali iodide salts. a.i., absolute intensity. Panel A: $\mathrm{mass}$ spectrum of $20 \mathrm{mg} / \mathrm{mL} F 20 T P P$ + $4 \mathrm{mM} \mathrm{Lil,} \mathrm{Nal,} \mathrm{KI,} \mathrm{Rbl,} \mathrm{and} \mathrm{Csl.} \mathrm{No} \mathrm{free} \mathrm{lithium} \mathrm{ions} \mathrm{were} \mathrm{observed.} \mathrm{Panel} \mathrm{B:} \mathrm{mass} \mathrm{spectrum} \mathrm{of} 10 \mathrm{mg} / \mathrm{mL} \mathrm{DHB}+4 \mathrm{mM} \mathrm{Lil,} \mathrm{Nal}, \mathrm{KI}, \mathrm{Rbl}$, and Csl. No free lithium ions were observed.

the noise. The S/N threshold was set at 5 for the SNAP peak picking algorithm. Subsequently, a "search for masses" macro, kindly provided by Bruker Daltonics, was used to automatically extract in batch peak parameters (peak intensity, peak $\mathrm{S} / \mathrm{N}$, and peak area) for specific masses (analyte and internal standard) from the peak lists generated by the above-described FlexAnalysis script.

Precision and Accuracy Calculations. Full analysis was performed when the analyte and internal standard were present at $\mathrm{S} / \mathrm{N}>3$ or $>5$ (vide supra) in at least two out of the four replicates. Of each experiment, the consecutive series of concentration calibrators that fulfilled the FDA $\pm 20 / 15$ criteria for precision $^{14}$ was used for accuracy calculations, using the mean analyte-to-internal standard response ratios of the replicates of each calibrator. The consecutive series of calibrators was chosen in such way that it contained the calibrator with the lowest analyte

(14) FDA 2001 available at http://www.fda.gov/cder/guidance/4252fnl.pdf. 


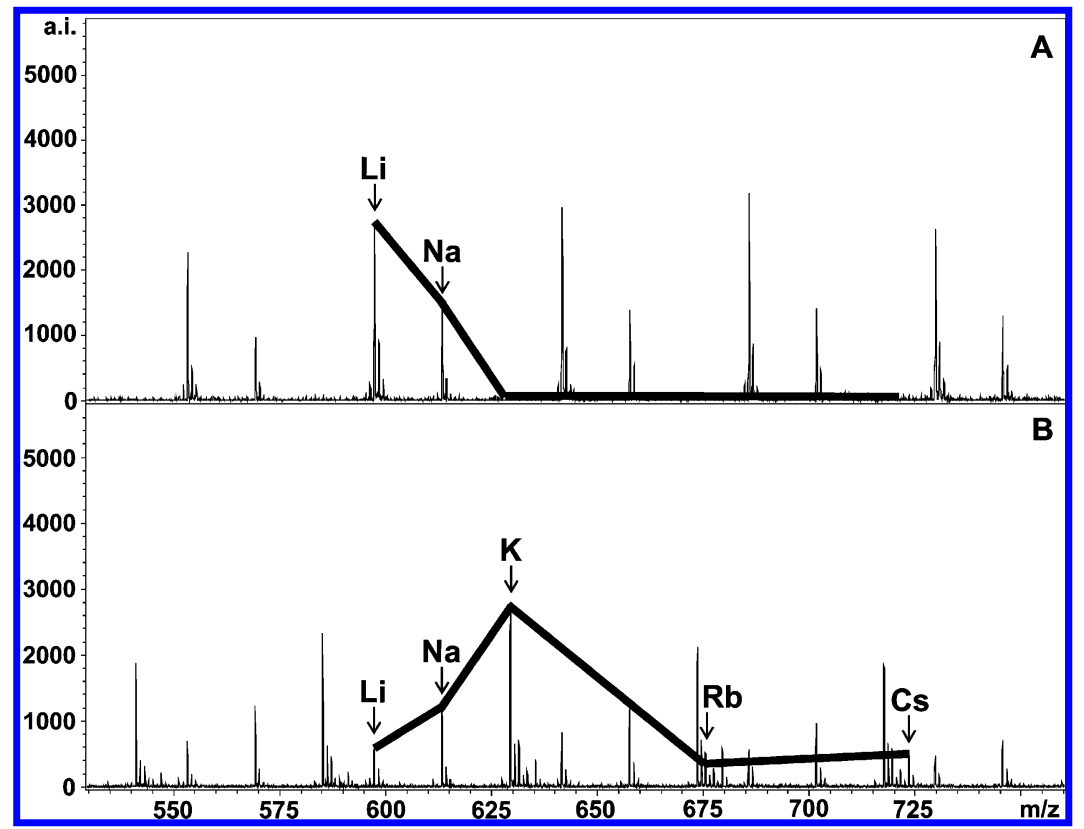

Figure 3. Distribution of cationized PEG600 using equimolar alkali iodide salts. a.i., absolute intensity. Panel A: mass spectrum of PEG600 analyzed with $20 \mathrm{mg} / \mathrm{mL}$ F20TPP $+4 \mathrm{mM}$ Lil, Nal, KI, RbI, and CsI. The most abundant signals were observed for lithiated PEG600, followed by sodiated PEG600. PEG600 did not form adducts with potassium, rubidium, or cesium. Panel B: mass spectrum of PEG600 analyzed with $10 \mathrm{mg} / \mathrm{mL}$ DHB $+4 \mathrm{mM}$ Lil, Nal, KI, Rbl, and Csl. The abundance of cationized PEG600 signals was as follows: $\mathrm{K}>\mathrm{Na}>\mathrm{Li}>\mathrm{Rb} \approx \mathrm{Cs}$. The solid line represents the cation distribution for PEG600 with a chain length of $13 \mathrm{CH}_{2} \mathrm{CH}_{2} \mathrm{O}$ units.

concentration. Unweighed, $1 / x$ weighed, and $1 / x^{2}$ weighed linear, and quadratic curve fitting methods were applied to construct the calibration curves. Calibrators with the highest analyte concentration of a consecutive series were removed one by one until that calibrator series fulfilled the FDA $\pm 20 / 15$ criteria for accuracy. ${ }^{14}$ Outliers were not removed unless otherwise stated.

\section{RESULTS AND DISCUSSION}

Meso-Tetrakis(pentafluorophenyl)porphyrin Matrix. The use of MALDI mass spectrometry for the quantitative analysis of drugs is normally hampered by interfering matrix peaks in the low-mass range and poor precision of signal abundances. ${ }^{1}$ Mesotetrakis(pentafluorophenyl)porphyrin (F20TPP) has a high molecular weight (MW 974.6 Da) compared to other more commonly used matrixes such as DHB (MW 154.1 Da) and $\alpha$-cyano-4hydroxycinnamic acid (MW 189.2 Da). Ayorinde et al. have shown that only a few interfering matrix peaks are observed in the lowmass range of F20TPP, which is prerequisite for the analysis of small molecules. ${ }^{7}$ To perform high-throughput quantitative analysis of drugs, we have developed a protocol for the rapid preparation of homogeneous F20TPP matrix crystals using prestructured target plates. F20TPP was dissolved in acetone and spotted on a hydrophobic target plate with hydrophilic anchors (AnchorChip) using a simple brushing. Each anchor absorbed $\sim 0.2 \mu \mathrm{L}$ of matrix solution, and homogeneous matrix crystals formed within $1 \mathrm{~s}$. The matrix can be spotted on all 384 anchors of a single target plate within $5 \mathrm{~min}$. Samples were spotted on top of the matrix crystals, and since F20TPP is not soluble in water, ethanol, or methanol, the sample solvent simply evaporates without perturbing matrix crystal homogeneity. Considering the good precision we can achieve (vide infra), we conclude that the analyte is indeed homogeneously incorporated into the matrix.

Upon laser irradiation, F20TPP forms radical cations and it also undergoes self-protonation. These protonated molecules may serve as proton donors for the analyte molecules. Our model compounds lopinavir and ritonavir contain peptide bonds, which have high proton affinities (PA). ${ }^{15}$ Thus, we expected that lopinavir and ritonavir would become protonated within the MALDI plume. However, no signals were observed for the protonated analytes when F20TPP was used as matrix (see Figure 1). Thus, F20TPP does not transfer a proton to the analyte, presumably because the PA of F20TPP is higher than that of lopinavir and ritonavir.

Another way to ionize molecules by MALDI is to add an alkali salt leading to alkali ion attachment. Indeed, for molecules lacking basic sites, such as sugars, cationization can be accomplished by the addition of alkali ions. ${ }^{3}$ This is also true when using the matrix F20TPP, which is usually mixed with sodium or potassium salts to ionize the analytes of interest. ${ }^{6-8,16}$ We investigated the effect of lithium, sodium, potassium, rubidium, and cesium iodide on the detection limits of lopinavir and ritonavir. Iodide was chosen as counteranion, because the lower lattice energy of alkali iodides compared to other alkali halides results in more free gaseous alkali ions and, thus, higher signal abundances of the cationized analytes. ${ }^{17}$ Considering the above argument based on lattice energies, it might reasonably be expected that for the alkali iodides the concentration of gaseous alkali ions will be in the order $\mathrm{Li}^{+}$ $<\mathrm{Na}^{+}<\mathrm{K}^{+}<\mathrm{Rb}^{+}<\mathrm{Cs}^{+}$. This is indeed observed. In Figure 2 are shown the MALDI-TOF mass spectra of equimolar amounts of the alkali iodides using F20TPP (Figure 2A) and DHB (Figure 2B) as matrix. As can be seen, the amount of gas-phase ions available for cationization is highest for $\mathrm{Cs}^{+}$, whereas virtually no lithium ions are liberated. On the other hand, the alkali affinity of

(15) Lias, S. G.; Bartmess, J. E.; Liebman, J. F.; Holmes, J. L.; Levin, R. O.; Maillard, W. G. J. Phys. Chem. Ref. Data 1988.

(16) Ayorinde, F. O.; Garvin, K.; Saeed, K. Rapid Commun. Mass Spectrom. 2000 14, 608-615.

(17) Hoberg, A. M.; Haddleton, D. M.; Derrick, P. J.; Jackon, A. T.; Scrivens, J. H. Eur. Mass Spectrom. 1998, 4, 435-440. 


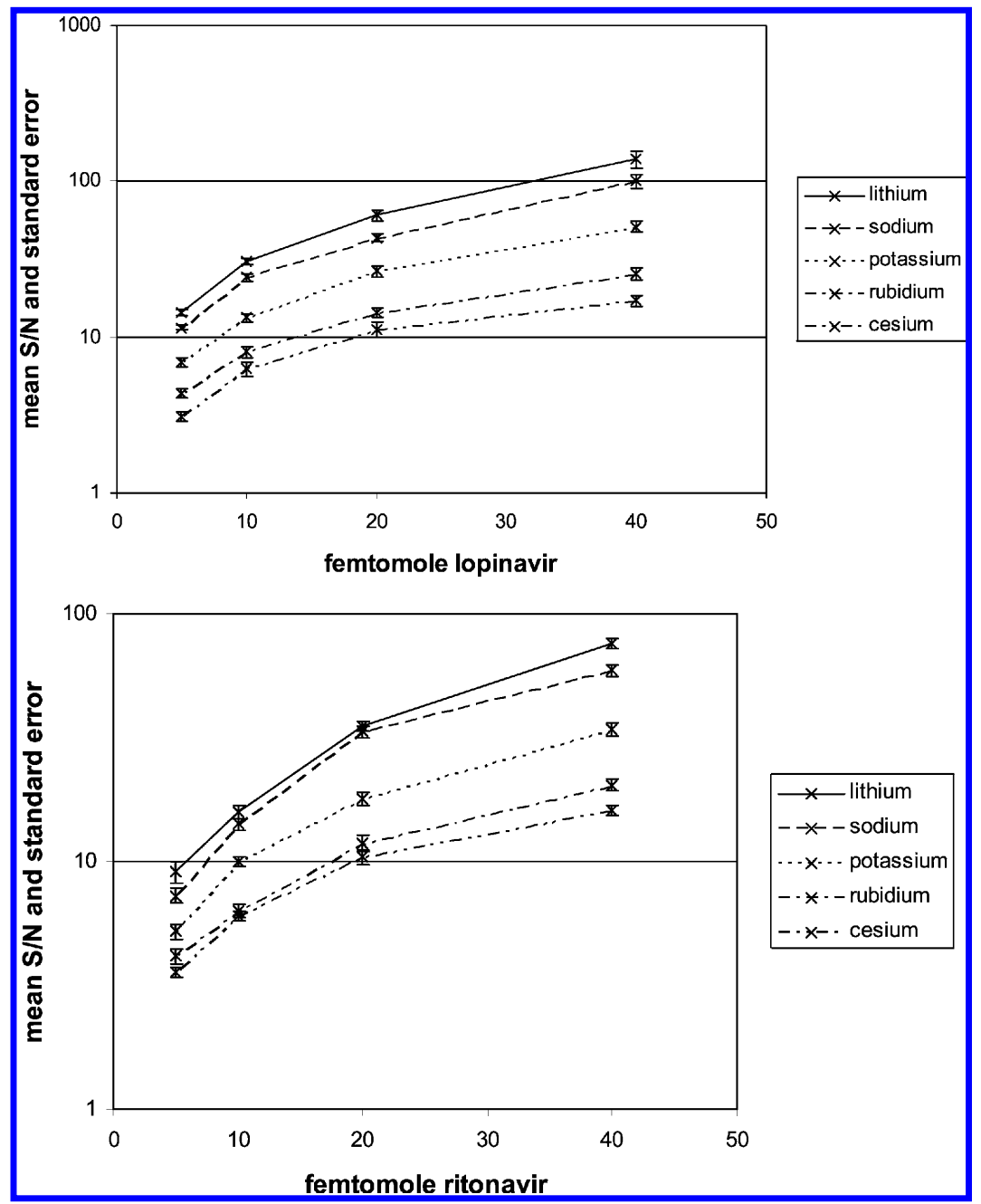

Figure 4. Effects of equimolar alkali iodide salts on the signal abundances of lopinavir and ritonavir $(n=12)$.

substrates will be in the opposite order, viz. $\mathrm{Li}^{+}>\mathrm{Na}^{+}>\mathrm{K}^{+}>$ $\mathrm{Rb}^{+}>\mathrm{Cs}^{+}$, because the smaller the ionic radius, the more stable the association of the alkali ion with the substrate will be (iondipole stabilization energies are inversely proportional to the square of the distance between the charge and substrate binding location). Thus, there are two opposing effects, to wit bare alkali ion availability and substrate alkali ion affinity. That there indeed are two opposing effects is borne out by cationization experiments using poly(ethylene glycol)s (PEGs) cationized by equimolar amounts of alkali iodides using DHB as matrix (see Figure 3B). An optimum for alkali attachment to the PEG chain was obtained for potassium in agreement with the operation of the two opposing effects discussed above. However, cationization experiments with PEGs and F20TPP showed a completely different distribution of the cationized PEG (see Figure 3A): An optimum alkali attachment to the PEG chain was obtained for lithium. Our two model compounds lopinavir and ritonavir showed a similar cation distribution when analyzed with F20TPP (Figure 4). The highest signal abundances for cationized lopinavir and ritonavir were observed when F20TPP was mixed with lithium iodide. We therefore conclude that alkali cationization using the F20TPP matrix does not occur by attachment of free lithium ions in the gas phase. This is in sharp contrast to results obtained with low molecular weight matrixes, such as $\mathrm{DHB}$, where free cation attachment is the predominant process. ${ }^{18}$ The mechanism of alkali attachment to pharmaceutical compounds using the matrix F20TPP is currently under investigation in our laboratories. For now, we will exploit the observation that lithium attachment produces the most intense signals. The combination of F20TPP and lithium iodide was therefore used as matrix for all other experiments.

Qualitative Analysis of Pharmaceutical Compounds. We obtained the detection limits $(\mathrm{S} / \mathrm{N} \geq 3)$ for the lithiated monoisotopic peaks of 26 drugs using the F20TPP matrix. Most of the drugs tested in this experiment are used to treat HIV or other infectious diseases. In addition, we tested drugs that are frequently prescribed or used to treat neurological diseases. The best detection limits were obtained for the HIV protease inhibitors lopinavir, ritonavir, saquinavir, indinavir, and nelfinavir (5 fmol). The HIV protease inhibitors amprenavir and tipranavir were detected up to 10 and $40 \mathrm{fmol}$, respectively. Erythromycin (10 fmol), omeprazole (10 fmol), carbamazepine (40 fmol), sulfamethoxazole $(40 \mathrm{fmol})$, and levofloxacin $(40 \mathrm{fmol})$ were detected at levels comparable to that of the HIV protease inhibitors. The detection limits for the HIV reverse transcriptase inhibitors lamivudine, nevirapine, zidovudine, and abacavir ranged from 500 to $1000 \mathrm{fmol}$. Comparable detection limits were obtained for primaquine (500 fmol), metoprolol (500 fmol), acetylsalicylic acid

(18) Zhang, J.; Zenobi, R. I. Mass Spectrom. 2004, 39, 808-816. 
Table 1. Effects of Twelve Data Analysis Procedures on the Mean Precisions ( $\%$ CV) of All Calibrators of Lopinavir and Ritonavira

\author{
procedures
}

monoisotope no intensity

monoisotope yes intensity

monoisotope no $\mathrm{S} / \mathrm{N}$

monoisotope yes $\mathrm{S} / \mathrm{N}$

monoisotope no area

monoisotope yes area

all isotopes no intensity

all isotopes yes intensity

all isotopes no $\mathrm{S} / \mathrm{N}$

all isotopes yes $\mathrm{S} / \mathrm{N}$

all isotopes no area

all isotopes yes area

LPV
11
12
11
12
8
9
19
15
19
18
13
11

RTV

10
10
10
10
9
9
9
9
9
9
9
9

${ }^{a}$ LPV, lopinavir. RTV, ritonavir. The left column shows which analyte signal was used (monoisotope, all isotopes), whether a baseline subtraction was used or not (yes/no), and which peak parameter was used (intensity, $\mathrm{S} / \mathrm{N}$, area).

(500 fmol), amikacin (1000 fmol), and tobramycin (1000 fmol). Ampicillin could be detected up to $4000 \mathrm{fmol}$, and acetaminophen, trimethoprim, penicillin $\mathrm{G}$, and rifampicin could not be detected using the F20TPP matrix.

Quantitative Analysis of Pure Lopinavir and Ritonavir. In the life sciences, MALDI-TOF mass spectrometry is mostly used for qualitative analysis of compounds such as peptides and proteins. As a consequence, little is known as to how data should be treated for quantitative analysis of small molecules. Therefore, we tested the effect of baseline subtraction, choice of peak parameter (intensity, $\mathrm{S} / \mathrm{N}$, area), and choice of analyte signal (monoisotope, all isotopes) on the precision of the lopinavir and ritonavir abundances. The results are shown in Table 1. For each entry, 16 concentrations of lopinavir and ritonavir were measured in 4-fold using indinavir as internal standard, and the average precision was calculated together with the standard deviation. The above procedure was performed three times, and from these three experiments, the average precision and standard deviation was extracted. Thus, the entries in Table 1 are the result of 192 measurements. The peak parameter "area" resulted in slightly better precisions of the lopinavir and ritonavir abundance compared to the peak parameter "intensity" or "S/N". Baseline subtraction did not have an effect on the precision. It can be seen that for lopinavir the precision deteriorates significantly when all isotopes are used. By contrast, for ritonavir no significant changes were observed. The poor precision of lopinavir when signal abundances of all isotopes were used may well be due to contamination of other compounds leading to interferences under the isotopic peaks of lopinavir.

Subsequently, we investigated whether drugs could be quantified by MALDI-TOF mass spectrometry according to the \pm 20 / 15 criteria of the FDA for precision and accuracy: Precision (expressed as relative standard deviation) and accuracy (expressed as \% deviation from the theoretical concentration) should be $\leq 15 \%$ for all calibrators except for the lower limit of quantification (LLOQ), which should be $\leq 20 \%{ }^{14}$ Table 2 shows the effects of baseline subtraction, choice of peak parameter (intensity, S/N, area), and choice of analyte signal (monoisotope, all isotopes) on the dynamic range of the lopinavir and ritonavir calibration curves
Table 2. Effects of Twelve Data Analysis Procedures on the Mean Dynamic Range of Lopinavir and Ritonavira

procedures
monoisotope no intensity
monoisotope yes intensity
monoisotope no S/N
monoisotope yes S/N
monoisotope no area
monoisotope yes area
all isotopes no intensity
all isotopes yes intensity
all isotopes no S/N
all isotopes yes S/N
all isotopes no area
all isotopes yes area

RTV
33
53
56
53
74
74
33
32
50
53
53
50

LPV

${ }^{a}$ RTV, ritonavir. LPV, lopinavir. The left column shows which analyte signal was used (monoisotope, all isotopes), whether a baseline subtraction was used or not (yes/no), and which peak parameter was used (intensity, S/N, area). Calibrator series of lopinavir and ritonavir were analyzed in 4-fold, and experiments were repeated three times. Subsequently, data were analyzed using 12 data analysis procedures (left column of the table), and precisions were calculated for the lopinavir and ritonavir calibrators. Calibrators that fulfilled the FDA \pm $20 / 15$ criteria for precision were used to construct calibration curves in the following way: six curve fitting methods were applied and calibrators with the highest analyte concentration were removed one by one until the calibration curve fulfilled the FDA $\pm 20 / 15$ criteria for accuracy. Reported here is the mean of the all dynamic ranges obtained by applying various curve fitting methods for each of the 12 data analysis procedures.

\section{Table 3. Effects of Six Curve Fitting Methods on the} Mean Dynamic Range Lopinavir and Ritonavira

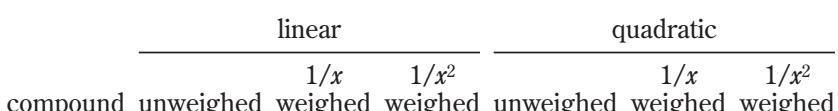

$\begin{array}{lllllll}\text { ritonavir } & 25 & 50 & 54 & 41 & 69 & 69 \\ \text { lopinavir } & 16 & 41 & 54 & 30 & 59 & 76\end{array}$

${ }^{a}$ Linear unweighed, linear $1 / x$ weighed, linear $1 / x^{2}$ weighed, quadratic unweighed, quadratic $1 / x$ weighed, and quadratic $1 / x^{2}$ weighed curve fitting methods were used to construct lopinavir and ritonavir calibration curves. Here we report the mean dynamic range of all 12 data analysis procedures (see Table 2) for each curve fitting method.

that fulfilled the FDA $\pm 20 / 15$ criteria for precision and accuracy. In general the best, i.e., largest, dynamic ranges were obtained by applying no baseline subtraction and by using the monoisotopic peak area. The application of baseline subtraction when the monoisotopic peak abundance of lopinavir was used resulted in a marked decrease in dynamic range. This effect was attributed to the increase in LLOQ from 1 (no baseline subtraction) to $5 \mathrm{fmol}$ (baseline subtraction). This effect was not observed for ritonavir. In general, the LLOQ increased when the signal abundances of all isotopes were used compared to the use of the monoisotopic peak abundance.

Table 3 shows the effects of the six curve fitting methods on the mean dynamic range of lopinavir and ritonavir. Quadratic curve fitting resulted in a larger dynamic range compared to linear curve fitting. Furthermore, the effect of weighing on the dynamic range, ordered from best to worst, was $1 / x^{2}$ weighing $>1 / x$ weighing $>$ unweighed curve fitting. The FDA states that the simplest model that accurately describes the concentration-response relationship 


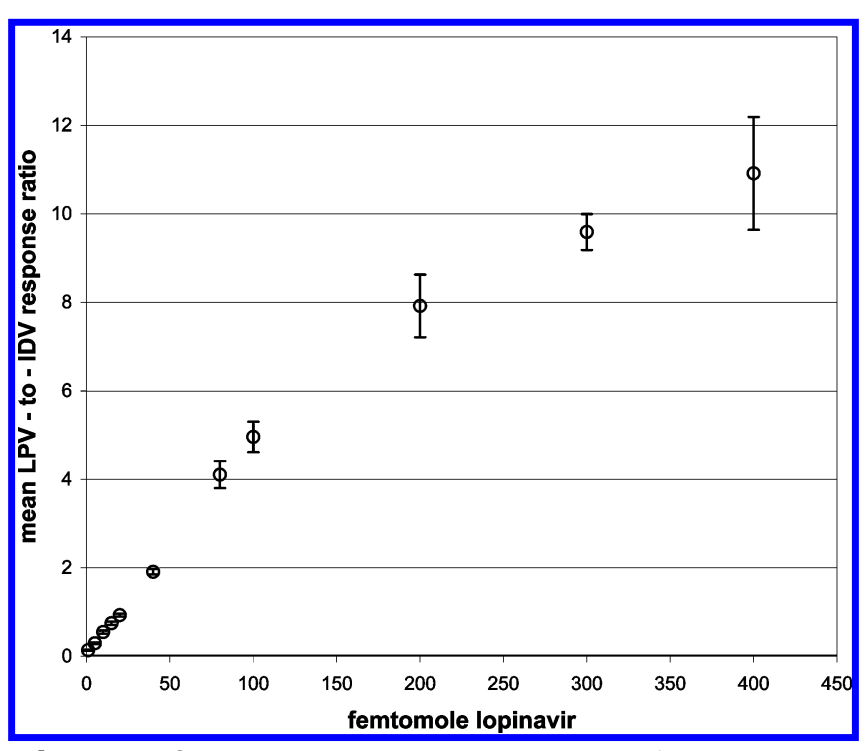

Figure 5. Concentration-response relationship of lopinavir using MALDI-TOF mass spectrometry. LPV, lopinavir; IDV, indinavir. Vertical bars represent plus and minus one standard deviation.

should be used to construct a calibration curve. ${ }^{19}$ In addition, the selection of weighing and use of complex regression equation should be justified, ${ }^{19}$ which we do here. MALDI-TOF mass spectrometers are typically equipped with 8-bit analog-to-digital converters $(\mathrm{ADC})$. These $\mathrm{ADCs}$ convert the intensity of the detector output into a numerical value of $0-255 .{ }^{20}$ Therefore, the dynamic range of MALDI-TOF mass spectrometers is generally limited to 2 orders of magnitude. Indeed, for our MALDI-TOF mass spectrometer, the concentration-response relationship was approximately linear when relative low concentrations of lopinavir were analyzed (up to $100 \mathrm{fmol}$ ), while the concentration-response relationship was more quadratic for higher lopinavir concentrations (see Figure 5). Furthermore, weighing functions were applied to reduce the influence of the higher concentrations on the fitted line, since the variance of the instrument response increases with the analyte concentration. ${ }^{19}$ In conclusion, the best dynamic ranges were obtained using the following data analysis procedure: No baseline subtraction, the use of the monoisotopic peak area, and construction of a calibration curve using a $1 / x^{2}$ weighed quadratic curve fitting method. Table 4 shows the precisions and accuracies of lopinavir and ritonavir when using this data analysis procedure.

Quantitative Analysis of Lopinavir in Cell Extracts. Next, we investigated whether MALDI-TOF mass spectrometry could be used to quantify clinically relevant concentrations of lopinavir in PBMC extracts according to the FDA $\pm 20 / 15$ criteria for precision and accuracy. Crommentuyn et al. reported a mean intracellular lopinavir concentration in 11 HIV-1 infected adults of $6.4 \mathrm{pmol} / 1 \times 10^{6} \mathrm{PBMCs}$ just before drug intake, and the peak concentration was $8.5 \mathrm{pmol} / 1 \times 10^{6} \mathrm{PBMCs} .{ }^{21}$ The results of our

(19) Garofolo, F. In Analytical method validation and intstrument performance verification.; Chan, C. C., Lam, H., Lee, Y. C., Zhang, X. M., Eds.; John Wiley \& Sons: New York, 2004; pp 105-138.

(20) In Mass spectrometry: A textbook; Gross, J. H., Ed.; Springer: New York, 2004; pp 113-130.

(21) Crommentuyn, K. M.; Mulder, J. W.; Mairuhu, A. T.; van Gorp, E. C.; Meenhorst, P. L.; Huitema, A. D.; Beijnen, J. H. Antiviral Ther. 2004, 9 , $779-785$.
Table 4. Quantitative Analysis of Pure Lopinavir and Ritonavira

\begin{tabular}{|c|c|c|c|c|c|c|}
\hline & \multicolumn{3}{|c|}{ lopinavir } & \multicolumn{3}{|c|}{ ritonavir } \\
\hline & $\exp 1$ & $\exp 2$ & $\exp 3$ & $\exp 1$ & $\exp 2$ & $\exp 3$ \\
\hline LLOQ (fmol) & 1 & 1 & 1 & 5 & 5 & 15 \\
\hline ULOQ (fmol) & 200 & 300 & 500 & 900 & 500 & 700 \\
\hline mean precision & $6.3 \pm 2.0$ & $6.8 \pm 3$ & $6.1 \pm 3.9$ & $7.0 \pm 3$ & $7.7 \pm 3$ & $7.3 \pm 4.6$ \\
\hline
\end{tabular}

${ }^{a}$ LLOQ, lower limit of quantification. ULOQ, upper limit of quantification. exp, experiment. Calibrator series of pure lopinavir and ritonavir were measured in 4 -fold, and experiments were repeated three times. Precisions of the mean analyte-to-internal standard response ratios for each calibrator were calculated using the monoisotopic peak areas. Calibrators that fulfilled the FDA $\pm 20 / 15$ criteria for precision were used to construct calibration curves in the following way: A $1 / x^{2}$ quadratic curve fitting method was applied, and calibrators with the highest analyte concentration were removed one by one until the calibration curve fulfilled the FDA $\pm 20 / 15$ criteria for accuracy. The reported precisions and accuracies are the mean values for all calibrators that fulfilled the $\pm 20 / 15$ criteria for precision and accuracy.

\section{Table 5. Quantitative Analysis of Lopinavir in PBMC} Extractsa

\begin{tabular}{llll} 
& \multicolumn{1}{c}{$\operatorname{exp~1}$} & \multicolumn{1}{c}{$\operatorname{exp~2}$} & \multicolumn{1}{c}{$\operatorname{exp~3}$} \\
LLOQ (fmol) & 25 & 25 & 25 \\
ULOQ (fmol) & 4000 & 3000 & 2000 \\
mean precision & $7.4 \pm 3.7$ & $8.4 \pm 4.8$ & $6.2 \pm 3.4$ \\
mean accuracy & $5.5 \pm 5.3$ & $5.7 \pm 4.6$ & $4.9 \pm 4.4$
\end{tabular}

${ }^{a}$ LLOQ, lower limit of quantification. ULOQ, upper limit of quantification. exp, experiment. Processed calibrator series were measured in 4-fold, and experiments were repeated three times. The mean analyte-to-internal standard responses were calculated for each calibrator using the monoisotopic peak areas. Calibration curves were constructed using a $1 / x^{2}$ weighed quadratic curve fitting. The reported precisions and accuracies are the mean values for all calibrators that fulfilled the FDA $\pm 20 / 15$ criteria for precision and accuracy. In experiment 3 , one outlier was removed.

experiments are shown in Table 5. As can be seen, our procedure allows the quantification of lopinavir down to $25 \mathrm{fmol}$ in extracts of $1 \times 10^{6}$ PBMCs according to the FDA $\pm 20 / 15$ criteria for precision and accuracy. This is well below the clinically relevant concentrations mentioned above. These results clearly show that MALDI-TOF mass spectrometry can be successfully used for clinical studies on the intracellular pharmacology of lopinavir. In Figure 6 are shown the mass spectra of PBMC extracts. It can be seen that after solid-phase extraction the spectra are remarkably clean. Considering that the mean signal-to-noise ratio $(n=12)$ obtained at the LLOQ was 9 (sd 2.3), better quantification limits seem possible.

In clinical studies on the intracellular pharmacology of antiretroviral drugs, aqueous mixtures containing a high percentage of organic solvent are used to extract the antiretroviral drugs from the PBMCs. We therefore prepared calibrator series of lopinavir in ethanol/water (1:1), and these calibrators were used to extract $1 \times 10^{6}$ PBMCs. One million PBMCs were used for these experiments, since this amount of PBMCs can be obtained from a blood sample of $1-2 \mathrm{~mL}$, a prerequisite for clinical studies in HIV-1 infected children.

A time-of-flight mass analyzer is normally not the first choice for quantitative analysis of drugs. As described above, the dynamic range is relatively small due to the use of analog-to-digital 


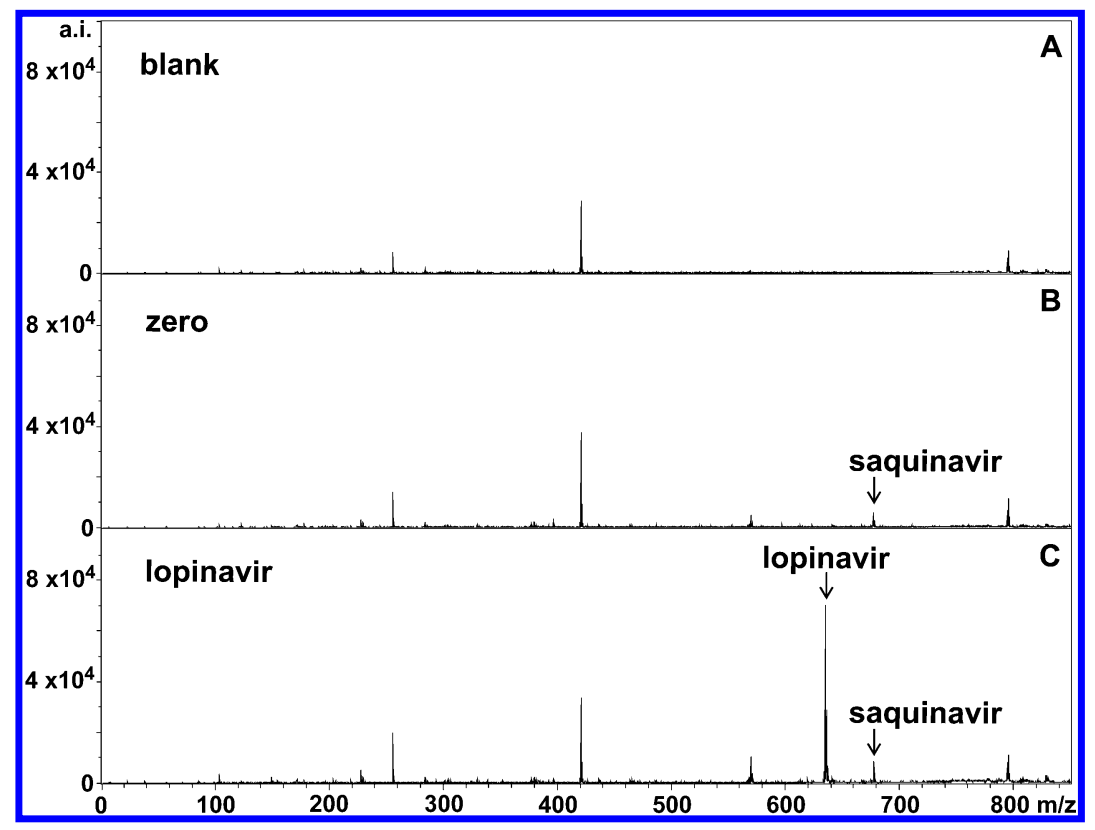

Figure 6. Mass spectra of PBMC extracts analyzed by MALDI-TOF mass spectrometry. a.i., absolute intensity. Panel A: mass spectrum of $1 \times 10^{6}$ PBMCs that were extracted using ethanol/water without adding drugs. Panel B: mass spectrum of $1 \times 10^{6}$ PBMCs that were extracted using ethanol/water spiked with internal standard only (500 fmol $/ \mu \mathrm{L}$ saquinavir). Panel C: mass spectrum of $1 \times 10^{6}$ PBMCs that were extracted using ethanol/water spiked with internal standard (500 fmol/ $\mu \mathrm{L}$ saquinavir) and analyte ( $2000 \mathrm{fmol} / \mu \mathrm{L}$ lopinavir).

converters with an 8-bit size. Furthermore, most time-of-flight analyzers are not designed to perform tandem mass experiments (MS/MS) on small molecules in contrast to, for example, triple quadrupole mass analyzers. Tandem mass experiments are used to increase signal-to-noise ratios and selectivity of drug measurements. Therefore, the development of new types of MALDI mass spectrometers, such as MALDI-triple quadrupole mass spectrometers, is of special interest for the quantitative analysis of drugs. ${ }^{12,22-24}$

Sample volumes typically used in MALDI mass spectrometry are in the order of $1 \mu \mathrm{L}$. The challenge for the successful application of MALDI mass spectrometry for quantitative analysis of drugs is to concentrate analytes in such a small volume in a reproducible manner. In our experiment, we reconstituted the processed calibrators in $100 \mu \mathrm{L}$ of ethanol/water, but only $1 \mu \mathrm{L}$ was spotted on to the target plate. Thus, a factor 100 in sensitivity was lost. Nevertheless, the obtained quantification limit of at least $25 \mathrm{fmol}$ for lopinavir in cell extracts allows reliable and clinical applications of our procedure. However, loss in sensitivity of a factor 100 could complicate the quantitative analysis of other drugs that have lower intracellular concentrations than lopinavir. To circumvent this problem, smaller solvent volumes could be used to reconstitute calibrators after vacuum-drying. Another way is to elute calibrators in a smaller volume from the solid-phase extraction plate and to directly spot the eluents onto the target plate. For example, Gobey et al. eluted calibrators in volumes of $25 \mu \mathrm{L}$ and spotted the eluents directly onto a target plate prior to quantitative analysis using a MALDI-triple quadrupole mass spectrometer. ${ }^{22}$

(22) Gobey, J.; Cole, M.; Janiszewski, J.; Covey, T.; Chau, T.; Kovarik, P.; Corr, J. Anal. Chem. 2005, 77, 5643-5654.

(23) Sleno, L.; Volmer, D. A. Rapid Commun. Mass Spectrom. 2005, 19, 19281936.

(24) Sleno, L.; Volmer, D. A. Anal. Chem. 2005, 77, 1509-1517.
One of the hallmarks of MALDI mass spectrometry is the speed of analysis. On our MALDI-TOF mass spectrometer, a typical time to analyze a sample in 4-fold in an automated way was 2 min and $20 \mathrm{~s}$. Our MALDI-TOF mass spectrometer is equipped with a $50-\mathrm{Hz}$ nitrogen laser, and the use of higher repetition rate lasers could further decrease analysis time to a few seconds as described by Volmer and co-workers. ${ }^{12}$ Fast sample preparation techniques are needed to exploit the high-throughput capacity of MALDI mass spectrometers. To this end, we have used 96-well solid-phase extraction plates allowing fast sample preparation. In addition, our pipetting robot is compatible with the 96well format, allowing fast, automated, and reproducible sample spotting. Furthermore, using the described brushing, the matrix can be spotted onto the target plate in $5 \mathrm{~min}$.

\section{CONCLUSIONS}

We have constructed a protocol by which pharmaceutical drugs, especially HIV protease inhibitors, can be successfully quantified in peripheral blood mononuclear cells using MALDITOF mass spectrometry. We have examined various acquisition procedures and postacquisition processing parameters. Crucial for acquiring reliable data is the use of a high molecular weight matrix, thereby virtually eliminating chemical noise, and the use of $\mathrm{Li}^{+}$to achieve efficient cationization of the drug. The choice of postacquisition data analysis procedures has a profound effect on the quantitative performance of the MALDI-TOF mass spectrometry technique. The best results were obtained using the monoisotopic peak areas of the analyte and internal standard, weighed quadratic curve fitting to construct the calibration curves, and no baseline subtraction. Using our protocol, we were able to quantify clinically relevant concentrations of lopinavir in cell extracts according to the $\pm 20 / 15$ criteria of the FDA for precision and accuracy. 


\section{ACKNOWLEDGMENT}

We gratefully acknowledge Aids Fonds, The Netherlands (project 2004051) and VIRGO for their financial support. Antiretroviral drugs were kindly provided by Abbott Laboratories, Merck Sharp \& Dohme, F.Hoffmann-La Roche, Pfizer Inc., GlaxoSmithKline, and Boehringer Ingelheim. We thank Arndt Asperger (Bruker Daltonik) for showing us the how to prepare thin matrix layers on an AnchorChip target plate, and Sören-Oliver Deininger (Bruker Daltonik) for providing the FlexAnalysis scripts. Conflicts of interest: The authors have a patent pending on the use of mesotetrakis(pentafluorophenyl)porphyrin in combination with lithium compounds for MALDI mass spectrometry.

Received for review March 9, 2006. Accepted May 17, 2006.

AC060436I 